Results Mean age of the participants was 30.5 years, $46 \%$ were nulliparous, $6.3 \%$ received fertility treatment, mean BMI was 23.6 $\mathrm{kg} / \mathrm{m}^{2}$. Prevalence of sickness absence until pregnancy week 30 was $36 \%$. Preliminary results indicate that sickness absence is related to fertility treatment and obesity. Women receiving fertility treatment had increased odds of sickness absence in pregnancy week 30; OR: 1.31 (95\% CI: 1.21-1.42). Obese women had increased odds of sickness absence compared to normal weight women; OR: 1.37 (95\% CI: 1.28-1.48). More statistical analyses will be conducted. Conclusions Final results and conclusions will be presented at the conference.

\section{THE USE OF EPIDEMIOLOGIC DATA TO EVALUATE THE ECONOMIC BURDEN OF OCCUPATIONAL RISKS: MODELLING THE COST OF DISEASES ATTRIBUTABLE TO JOB STRAIN IN FRANCE}

1,2 Hélène Sultan-Taïeb, 3,4 Jean-François Chastang, ${ }^{5}$ Malika Mansouri 3,4 4 sabelle Niedhammer. 'Université Du Québec A Montréal (UQAM), Montréal, Québec, Canada; ${ }^{2}$ Centre de Recherche Interdisciplinaire Sur Le Bien-Etre, La Santé, La Société Et L'environnement (CINBIOSE), Montréal, Québec, Canada; ${ }^{3}$ INSERM, U1018, CESP Centre for Research in Epidemiology and Population Health, Epidemiology of Occupational and Social Determinants of Health Team, Villejuif, France; ${ }^{4}$ Laboratoire D'Économie Et Gestion, Université de Bourgogne, Dijon, France; ${ }^{5}$ Université de Versailles St-Quentin, UMRS 1018, Versailles, France

\subsection{6/oemed-2014-102362.62}

Objectives To estimate the annual costs of coronary heart diseases (CHD) and mental disorders (MD) attributable to job strain exposure according to Karasek's model in France for the year 2003 from a societal perspective.

Method We produced attributable fraction estimates which were applied to the number of cases (morbidity and mortality) and the costs of CHD and MD. Relative risk estimates came from a systematic literature review of prospective studies. We conducted meta-analyses based on this selection of studies. Prevalence of exposure to job strain came from the national SUMER survey conducted in France in 2003.

Results Between 8.8 and 10.2\% of CHD morbidity and between 9.4 and $11.2 \%$ of CHD mortality was attributable to job strain for men. Between 15.2 and $19.8 \%$ of MD was attributable to job strain for men, and between 14.3 and $27.1 \%$ for women. The total costs of $\mathrm{CHD}$ and $\mathrm{MD}$ attributable to job strain exposure ranged from 1.8 to 3 billion euros for the year 2003 (0.12-0.19\% GDP). Medical costs accounted for $11 \%$ of the total costs, value of life costs accounted for $13-15 \%$ and sick leave costs for $74-77 \%$. The cost of CHD was estimated at 113-133 million euros and the cost of MD was between 1.7-2.8 billion euros in 2003.

Conclusions This study on the economic burden of diseases attributable to job strain in France provides relevant insights for policy-makers when defining public health priorities for prevention policies.

\section{PREVALENCE OF OCCUPATIONAL EXPOSURE TO LEAD IN AUSTRALIA}

${ }^{1}$ Tim Driscoll, ${ }^{2}$ Renee Carey, ${ }^{3}$ Deborah Glass, ${ }^{3}$ Geze Benke, ${ }^{2}$ Susan Peters, ${ }^{2}$ Alison Reid, ${ }^{2}$ Lyn Fritschi. 'Sydney School of Public Health, University of Sydney, Sydney, NSW, Australia; ${ }^{2}$ Western Australian Institute for Medical Research, University of Western Australia, Perth, WA, Australia; ${ }^{3}$ Department of Epidemiology and Preventive Medicine, Monash University, Melbourne, VIC, Australia

10.1136/oemed-2014-102362.63
Objectives To determine the prevalence of work-related exposure to lead, the main circumstances of work-related exposure to lead in the general workforce, and the use of workplace control measures designed to decrease exposure to lead, in Australia.

Method The information came from the Australian Work Exposures Study (AWES) project, a nationwide survey which investigated the current prevalence of work-related exposure to 38 known or suspected carcinogens, including lead, among Australian workers, based on reported job tasks. Only those persons designated as having probable work-related exposure to lead were included in the analysis. Assessments were extrapolated to the national workforce with reference to the 2011 Census.

Results The results suggest approximately $6.6 \%$ of Australian workers were occupational exposed to lead. Almost all exposed workers were male, about half workers worked in technical occupations and almost half worked in the construction industry. The main tasks associated with probable exposures were, in decreasing order, soldering; painting old houses, ships or bridges; plumbing work; cleaning up or sifting through the remains of a fire; radiator repair work; machining metals or alloys containing lead; mining; and welding leaded steel. The use of appropriate respiratory control measures was inconsistent. Exposure levels were assessed as being high or medium in most cases, taking into account information on work tasks and the controls being used by workers.

Conclusions The study suggests exposure to lead in the Australian workforce is higher than expected based on estimates from other countries. There is considerable scope for better use of exposure control measures.

\section{JOB STRAIN AND BURNOUT AMONG NURSES WORKING IN DIFFERENT HEALTHCARE SETTING}

${ }^{1}$ Weishan Chin, ${ }^{2}$ Li-Jie Wang, ${ }^{3} J u d i t h$ Shu-Chu Shiao, 1,2Yue-Liang Leon Guo Guo, ${ }^{1}$ Shan-wei Yang. Institute of Occupational Medicine and Industrial Hygiene, National Taiwan University School of Public Health, Taipei, Taiwan; ${ }^{2}$ Department of Environmental and Occupational Medicine, National Taiwan University and NTU Hospital, Taipei, Taiwan; ${ }^{3}$ Department of Nursing, College of Medicine, National Taiwan University (NTU) and NTU Hospital, Taipei, Taiwan; ${ }^{4}$ Department of Environmental and Occupational Medicine, National Taiwan University Hospital, Taipei, Taiwan

\subsection{6/oemed-2014-102362.64}

Objectives To assess job strain and burnout status among female nurses working in primary clinics, secondary referral hospitals, and public health units in Taiwan.

Method Study participants included female nurses from (1) all primary clinics (PC) hiring more than two registered nurses; (2) a nation-wide representative sample of secondary referral hospitals (SRH), selected using stratified random sampling; and (3) all public health units (PHU) hiring more than two registered nurses. To candidate participants, a structured, self-administered questionnaire was disseminated, which included demographic information, work conditions, the Chinese Job Content Questionnaire, and the modified Chinese Copenhagen Burnout Inventory.

Results A total of 6087 questionnaires were sent, and 4046 $(66.5 \%)$ were satisfactorily completed. Compared with PC nurses, nurses working in SRHs and PHUs had higher job strain (adjusted odds ratio, $\mathrm{aOR}=1.7,95 \%$ confidence interval, $\mathrm{CI}=1.3-2.1$ for $\mathrm{SRH}$; $\mathrm{aOR}=2.4,95 \% \mathrm{CI}=1.7-3.4$ for $\mathrm{PHU}$ ), personal burnout $(\mathrm{aOR}=2.6,95 \% \mathrm{CI}=1.8-3.6$ for $\mathrm{SRH}$; $\mathrm{aOR}=3.4,95 \% \mathrm{CI}=2.1-5.7$ for $\mathrm{PHU}$ ), work-related burnout 